\title{
L'IMAGE À L'INFINI : \\ La lente accomodation de la profession enseignante \\ à une identité féminine
}

\section{António NOVOA*}

Écrire pour ne pas mourir, comme disait Blanchot, ou peut-être même parler pour ne pas mourir est une tâche aussi vieille sans doute que la parole. Les décisions les plus mortelles, inévitablement, restent suspendues le temps encore d'un récit. Le discours, on le sait, a le pouvoir de retenir la flèche, déjà lancée, en un retrait du temps qui est son espace propre (Michel Foucault, 1963).

Le discours éducatif est, avant tout, imagé. L'enseignement et l'éducation "sont choses dont on parle, choses qui se parlent, qui ne peuvent pas ne pas se parler" (Hameline, 1986, p. 15). Et les images sont le meilleur moyen pour en parler, car elles suggèrent l'action et constituent ainsi des éléments essentiels à une pensée pédagogique qui ne peut être purement spéculative ou praxéologique.

Les images font partie du monde des enseignants. Elles construisent des dispositifs d'adhésion (ou de rejet) qui configurent différents modèles professionnels. Elles sont porteuses de projets éducatifs, parfois contradictoires, et fonctionnent comme l'un des langages privilégiés pour se dire éducateur, pour définir une certaine manière d'être dans la profession.

\footnotetext{
* Université de Lisbonne.
} 
Dans ce texte ${ }^{1}$, je propose une interprétation large, étalée sur un siècle, sur les images comme lieux de construction sociale des enseignants. Il s'agit d'une réflexion libre, dans laquelle les images seront approchées comme des textes qui transmettent des visions conflictuelles, comme des espaces de pouvoir qui déterminent l'existence personnelle et professionnelle des enseignants. Elles ne seront pas envisagées comme des signes d'une quelconque réalité « cachée », mais, au contraire, comme des éléments qui font partie intégrante des pratiques discursives qui construisent cette même réalité (Nóvoa, 1995).

Je tiendrai un argument historique, en essayant de raconter à grands traits la lente accomodation de la profession enseignante à son identité féminine. Ma narrative s'organise autour de trois temps-forts de l'histoire des enseignants et de la production d'images sur leur profession : le tournant du XIX ${ }^{\mathrm{e}}$ siècle, le milieu du $\mathrm{XX}^{\mathrm{e}}$ siècle et la période actuelle. Je chercherai à présenter un point de vue - et ce n'est qu'un point de vue parmi beaucoup d'autres - portant sur les images masculines et féminines de la profession enseignante. La réflexion prend appui essentiellement sur un corpus iconographique concernant les instituteurs portugais, mais elle prétend rendre compte d'une idée qui, avec les nécessaires adaptations, peut être utile pour penser le parcours de différents groupes d'enseignants dans plusieurs contextes nationaux.

En effet, et sans nier que l'invention d'images a des spécificités nationales, il faut bien reconnaître qu'on est vis-à-vis un phénomème international. De l'un et de l'autre côté de l'Atlantique on retrouve les mêmes images, qui traduisent la ressemblance des dilemmes vécus par les enseignants, mais aussi la diffusion mondiale d'un même modèle scolaire (Meyer, Ramirez \& Soysal, 1992; Tyack \& Cuban, 1995). Le recueil d'images présenté par Ellwood Cubberley dans le Syllabus of Lectures on the History of Education

\footnotetext{
${ }^{1}$ Je reprends, pour l'essentiel, mon intervention dans la table ronde « Invention et diffusion d'images, dans la littérature, les médias, les sciences sociales » (INRP, 10 février 1995). J'ai décidé de garder le texte dans sa forme orale, tout en étant conscient qu'il s'agit de « réflexions provisoires » qui réclament des démonstrations postérieures.
} 
(1902), ainsi que le livre récent de Pamela Bolotin Joseph et Gail E. Burnaford, Images of Schoolteachers in Twentieth-Century America (1994), montrent bien que la circulation internationale des images sert à instituer une certaine vision de la profession enseignante. C'est pourquoi elles doivent être regardées comme des discours, et non pas comme de simples portraits du « réel ».

Aujourd'hui, l'historien ne cherche plus à restituer un passé unitaire qui serait inscrit dans les faits qu'il interroge ; bien au contraire, il essaye de comprendre toutes les histoires, en identifiant les groupes qui ont été investis de la légitimité sociale pour les raconter. C'est pourquoi mon analyse des images dans le temps privilégie les groupes qui ont détenu le pouvoir de les produire et de les diffuser, instaurant de la sorte une vision dominante des enseignants et de leurs processus individuels et collectifs d'identité professionnelle.

\section{L'affirmation publique et les images masculines des enseignants (fin du XIX ${ }^{\mathrm{e}}$ siècle)}

Je m'arrête d'abord à un temps-fort de l'affirmation publique des enseignants, la fin du $\mathrm{XIX}^{\mathrm{e}}$ siècle. Alors, le système éducatif assume la responsabilité majeure de la formation de citoyens intégrés dans le projet d'une nation qui se définit, le plus souvent, à l'intérieur des frontières d'un État souverain. Certains historiens parlent de l'État enseignant, pour bien marquer la liaison intime entre le «nouvel» État et le «nouvel» enseignant : il n'est pas possible de rendre raison de l'un sans faire référence à l'autre (et vice-versa). Les écoles normales constituent l'enceinte adéquate à discipliner les enseignants, devenus agents du projet social et politique de la modernité : les discours qui y sont produits édifient un modèle d'enseignant qui mélange les «anciennes» références religieuses avec le «nouveau» rôle de serviteurs de l'État. 
Les enseignants sont investis d'un important pouvoir symbolique, renforcé par la croyance dans un progrès social qui serait assuré par l'école. Dans ce contexte, ils n'acceptent plus un statut extrêmement détérioré du point de vue social et économique. La vision misérabiliste qui traverse traditionnellement l' « imagerie » des enseignants devient insupportable. Et c'est dans ce registre qu'il faut interpréter leur présence dans les caricatures publiées au tournant du XIX ${ }^{\mathrm{e}}$ siècle.

Dans un échantillon de plus de 200 caricatures d'enseignants ${ }^{2}$, j'ai choisi quatre exemples pour illustrer mon propos.

Le premier dessin, À la parade, est publié en 1886, à l'occasion d'une visite des souverains anglais au Portugal, dans une situation de grande tension politique entre les deux pays en vue du contrôle de plusieurs territoires africains.

Le caricaturiste suggère qu'on fasse défiler devant les anglais la redoutable armée des

\footnotetext{
${ }^{2}$ On n'a retenu que les caricatures publiées dans les années de transition du XIX ${ }^{\mathrm{e}}$ vers le XX ${ }^{\mathrm{e}}$ siècle. La recherche a été menée au Portugal, avec la collaboration de Vera Teixeira et Filomena Bandeira, ayant fait l'objet de l'édition d'un ensemble de cartes postales avec un texte d'introduction : Os professores e as reformas de ensino - Na viragem do século (1886-1906). Porto: Edições Asa.
} 
instituteurs, que l'on présentera ainsi : «-La meilleure et la moins chère des armées! Ils mangent, boivent, s'habillent, se chaussent et éduquent les citoyens pour un salaire de rien... payé uniquement dans les années bissextiles... ».

Le deuxième dessin,

Le paria, daté de 1887 ,

représente un

instituteur qui meurt

de faim, tandis que,

sur la droite, les

professeurs des

universités se

réjouissent d'une

nouvelle augmentation

salariale. Les éléments

iconographiques sont

tous là (la férule,

l'ABC, les lunettes,

etc.), illustrant

l'émergence d'un esprit

de corps qui se

construit contre

d'autres corps

professionnels.

S'esquissent, à cette

époque, les contours

identitaires de la

profession enseignante.

La troisième caricature, Le congrès des instituteurs, publiée en 1897, est particulièrement parlante. Les «misérables » deviennent menaçants, du fait de leur organisation collective : «Le prochain congrès des instituteurs à Porto sera le fléau de monsieur le Président du Conseil ». 
Les férules servent à frapper les mains du Premier ministre et à attaquer le « tronc des promesses » qui ne sont jamais tenues. Ce Congrès a été très important dans la consolidation du mouvement associatif des enseignants: des institutrices y ont participé activement, même dans les comités d'organisation, mais les images diffusées restent exclusivement masculines.

Finalement, la quatrième caricature, Le banquet, apparaît en 1906 pour confronter les « excès » des gouvernants

aux « manques » des

instituteurs. Elle serait

impensable quelques

années plus tôt,

car on met en

cause directement le

pouvoir de l'État.

Après de longues

revendications,

les structures

associatives des

enseignants sont,

enfin, reçues par

le Directeur-Général

de l'Instruction

Publique. Face à une

table où l'on présente

la meilleure cuisine

française, l'instituteur

exclame :

«-Comment se fait-il qu'il y ait des gens qui mangent ainsi tous les jours !... ».

Ces images peuvent être analysées sous différents angles. Je n'en retiens qu'un seul : l'absence d'images publiques des institutrices. Or, vers 1900, les femmes sont déjà majoritaires au sein du corps enseignant primaire : plus de 50\% en France ou en Belgique ; plus de 60\% en Suède ou en Italie ; plus de 70\% en Angleterre ou aux États-Unis. Et, pourtant, les images dominantes sont toujours des images masculines, lesquelles semblent convenir mieux à l'affirmation professionnelle des enseignants. 
C'est vrai qu'il y a aussi des images féminines, mais elles sont souvent « privées » (l'institutrice avec ses élèves ou dans sa classe). Simultanément, il y a des idéologies de maternage qui se diffusent dans le champ éducatif, notamment grâce au travail d'une nouvelle génération de médecins et d'hygiénistes. Mais la conception de l'enseignement comme profession féminine n'apparaît qu'en plan secondaire, comme l'on peut confirmer par la discrimination salariale subie par les institutrices de la plupart des pays jusqu'au début du XX $\mathrm{XX}^{\mathrm{e}}$ siècle (Araújo, 1993).

L'image utile, notamment à la lutte syndicale et à la recherche d'une plus grande reconnaissance sociale, reste masculine. La dimension féminine de l'enseignement est acceptée, mais ne constitue pas la référence identitaire de la profession. Bien au contraire, ce sont les images masculines qui s'adaptent le mieux au modèle professionel dominant. À cette époque, ce qui colle à la peau des instituteurs relève, plutôt, d'une ideólogie de progrès qui renvoye à une intervention publique et à un combat socio-politique (ceci ne signifie pas que les institutrices n'interviennent pas sur le plan social et politique, notamment dans le cadre des mouvements féministes, mais elles le font d'une toute autre manière). Les enseignants se fabriquent eux-mêmes par des mots qui appartiennent fondamentalement au genre masculin. 


\section{La féminisation et les images " privées " $\underline{\text { des enseignants (milieu du } \mathrm{XX}^{\mathrm{e}} \text { siècle) }}$}

Après 1918, la profession enseignante vit un temps de transition, marqué de tensions provoquées par la critique à un modèle scolaire qui a été incapable d'empêcher l'horreur de la Guerre. Il fallait former un homme nouveau, mais pour cela besoin en était d'une école nouvelle et, a fortiori, d'un nouvel enseignant. Aux années vingt on assiste à l'émergence d'une nouvelle conception du travail pédagogique et de l'identité professionnelle des enseignants.

Il y a, d'une part, le discours de l'Éducation Nouvelle, qui est à beaucoup d'égards la conséquence pédagogique d'une évolution des moeurs et du rapport à l'enfance. Le fameux prologue d'Adolphe Ferrière à son ouvrage Transformons l'école, où il présente l'invention de l'école comme une création du Diable, illustre bien un changement qui va s'inscrire dans le champ pédagogique et dans la manière dont chacun se dit éducateur: au lieu de la férule, l'affect ; au lieu de la corvée, le jeu ; au lieu de l'obéissance, la créativité ; au lieu de la besogne, le plaisir ; au lieu du silence, la communication. Sans prolonger le manichéisme d'une approche qui oppose l'école traditionnelle à l'école nouvelle - et sans forcer la démonstration stéréotypée -, il faut bien reconnaître l'émergence d'un univers féminin dans la pensée éducationnelle. L'Éducation Nouvelle est porteuse d'images contrastantes sur les enseignants, mais on voit se dessiner une présence féminine, qui avait été absente (ou, en tout cas, silencieuse) jusque-là.

Il y a, d'autre part, la continuité d'une tendance envers la féminisation de la profession enseignante. Aux États-Unis, le pourcentage de femmes dans le corps enseignant primaire est environ 90\% au début des années trente. En Europe, les pourcentages sont plus bas, mais suffisamment significatifs pour que la question du genre se pose de façon accrue dans le processus identitaire des enseignants. On connaît les 
arguments classiques, qui cherchent à expliquer la féminisation à travers la dévalorisation économique et professionnelle de l'activité enseignante (et vice-versa). À la suite de Michael Apple (1988), il est peut-être plus intéressant de poser une autre question : Est-ce que nous ne sommes pas devant un changement essentiel du métier d'enseignant ? En effet, cette problématique ne peut pas être approchée par le biais d'un simple raisonnement statistique ou quantitatif, tout en étant nécessaire de la replacer dans un champ complexe d'influences et de projets contradictoires pour la profession enseignante.

Simultanément - et pour des raisons qui relèvent à la fois du politique, du social et du culturel - les enseignants vont être écartés, petit à petit, du devant de la scène publique. Dès les années trente, on a l'impression qu'un manteau de silence va tomber sur les enseignants, et les renfermer à l'intérieur des espaces scolaires. Ce retrait s'accompagne d'une rhétorique qui réinvente les concepts de mission, de sacerdoce ou de vocation, tout en les adaptant à l'univers féminin. Le métier d'enseignant s'accomode à une identité féminine et cela entraîne des images plutôt privées : les instituteurs sont souvent figurés sur des espaces publics, tandis que les institutrices tendent à être représentées à l'intérieur de l'enceinte scolaire. Comme si la mission de l'instituteur était définie par l'élargissement du scolaire vers le public (intervention sociale et politique) et celle de l'institutrice par le rétrécissement du scolaire vers le privé (action éducative et pédagogique).

En rapport avec cette transition, on assiste au renforcement des dispositifs de contrôle des enseignants, qui, surtout dans le cas des femmes, n'incident pas uniquement sur les aspects professionnels mais s'allongent sur les dimensions privées (Butler, 1993 ; Maynard \& Purvis, 1994). La vie et le corps des institutrices deviennent l'objet d'une surveillance accrue qui met en oeuvre des politiques discriminatoires vis-à-vis le travail féminin. Les conditions imposées aux femmes lors de la signature de leur contrat de travail comme enseignantes illustrent bien mon propos ; Michael Apple transcrit un contrat de 1923, tout en informant qu'aux États-Unis ce modèle s'est répandu « pendant 
plusieurs décennnies, ayant par la suite pris des formes plus techniques et bureaucratiques de contrôle » (1988, p. 74).

\footnotetext{
Voilà une image prégnante qui s'impose aux femmes enseignantes :
}

«Il serait inadéquat d'essayer d'expliquer cette situation uniquement par des motivations économiques ou des stratégies de classe. C'est vrai que les femmes célibataires sont souvent jeunes et moins payées, mais les notions de moralité et de pureté, en tant que symboles du travail enseignant féminin, doivent être prises en considération. Les dispositifs de contrôle du corps, la façon de s'habiller, les conditions de vie ou les comportements moraux des femmes montrent l'importance de ces symboles. On est face à la reproduction d'idéologies patriarcales - articulées, sans doute, avec une suspicion masculine à l'égard de la sexualité féminine - qui contribuent à entourer les enseignants d'un manteau domestique et maternel » (Apple, 1988, p. 72).

Cette image des enseignants domine les représentations (discours, écrits, photos, films...) au milieu du XX'e ${ }^{\mathrm{e}}$, ce qui n'empêche pas qu'elle soit concurrancée par d'autres images. On diffuse une illusion de stabilité, de « faux silence » sur les enseignants - dû, en grande mesure, à la production du mythe de la femme passive - qui tend à légitimer 
des lectures consensuelles de la profession. Je pense, par exemple, à un discours prononcé par Albert Shanker, leader syndical des enseignants nord-américains, en 1974:

"Toutes les années il y a 10000 nouveaux enseignants qui entrent dans le système scolaire de New York. Ils viennent d'un peu partout. Ils représentent toute sorte de religions, de races, de filiations politiques et même de formations professionnelles. Mais ce qui est très curieux c'est qu'après trois semaines en classe on ne les distingue plus les uns des autres » (cf. Cuban, 1983).

Cet appel à l'homogénéité de la profession enseignante doit être mis en rapport avec les tendances de rationalisation de l'enseignement qui se développent à partir des années soixante: à cette époque la recherche en sciences de l'éducation renforce le silence sur les enseignants. En effet, l'effort d'édification d'une pédagogie scientifique porte sur l'analyse « objective » du processus enseignement-apprentissage, de telle façon que l'enseignant ne constitue qu'une variable parmi beaucoup d'autres. Ce n'est pas un hasard si les recherches portant spécifiquement sur les enseignants ont considérablement diminué au cours des années soixante et soixante-dix, à l'exception des études sur la problématique de la formation (Cohn \& Kottkamp, 1993 ; Middleton, 1993).

La tentative de construire une narrative de l'enseignement en marge des enseignants contribue à entériner une définition technique du métier et, du même coup, à mettre en oeuvre des pratiques de contrôle basées sur des rationalités « scientifiques ». C'est le rapport des enseignants au savoir qui est directement mis en cause, à travers: la séparation entre la conception et l'application, ce qui entraîne une tutelle des experts sur les enseignants ; la standardisation des tâches, ce qui provoque une définition technique et instrumentale des enseignants ; l'intensification des activités scolaires quotidiennes, ce qui rend impossible une approche réflexive des pratiques pédagogiques (Nóvoa, 1994).

La rationalisation de l'enseignement met entre parenthèse les savoirs, les subjectivités, les expériences, en un mot, les histoires personnelles et collectives des enseignants. Se construit, ainsi, une logique de professionnalisation qui fait table rase des 
dimensions subjectives et expérientielles, des espaces de réflexion des enseignants sur leur propre travail, des moments informels d'échange et de coopération. Est-il étonnant que les enseignants, majoritairement des femmes, aient réagi contre ce modèle ? Est-ce que la crise d'identité des enseignants n'est pas liée à ce refus? 


\section{L'émergence d'images publiques et féminines des enseignants (fin du $\mathrm{XX}^{\mathrm{e}}$ siècle)}

Aux années quatre-vingts le thème de la « professionnalité enseignante » redevient à l'ordre du jour. Le livre coordonné par Ada Abraham, L'enseignant est une personne (1984), constitue une référence symbolique de l'effort tendant à créer de nouvelles références identitaires des enseignants, que l'on peut évoquer à partir de trois idées : la possibilité d'envisager la personne et son expérience comme critères de définition professionnelle ; la délimitation d'un espace d'intervention, c'est-à-dire l'organisation scolaire, comme niveau intermédiaire d'autonomie professionnelle; l'investissement des savoirs produits par les enseignants comme condition d'une nouvelle culture professionnelle (Nóvoa, 1994).

Ce changement ne doit pas être regardé uniquement sous l'angle du genre. Je suis d'accord avec Roger Chartier quand il met en garde contre le risque « d'investir la différence entre sexes d'une force explicative universelle » (1993, p. 1006). Néanmoins, cette voie d'explication doit être aussi prise en compte, et c'est pourquoi je soutiens que la nouvelle visibilité publique des enseignants peut être lue à la lumière de l'évolution du rôle social et de la pensée sur les femmes.

Je pose comme hypothèse polémique qu'après quelques décennies (au début du siècle) d'accomodation à une image féminine et après quelques décennies (au milieu du siècle) de gestion d'une subjectivité silencieuse, le métier d'enseignant retourne à l'espace public, mais cette fois-ci par l'affirmation d'une identité féminine. Le nouvel intérêt qu'on porte aux enseignants - très évident dans la littérature pédagogique et dans le travail scientifique à partir des années quatre-vingts - peut être analysé à partir de cette mutation historique. D'ailleurs, on constate sans surprise que l'agenda du féminisme est présente dans la réflexion actuelle sur les enseignants : on se réfère aux voix silencieuses, à la 
valorisation de l'expérience, à l'affirmation du privé, à l'importance de la subjectivité, à la reconstruction d'identités personnelles et professionnelles... (Canning, 1994 ; Casey, 1993 ; Gore, 1993 ; Luke \& Gore, 1992).

Il y a, sans doute, d'autres raisons qui expliquent le retour des enseignants sur la scène publique, à la fois dans la rhétorique politique, dans le discours scientifique et dans l'espace social de la profession. La rhétorique politique diffuse des images d'autonomie au moment même où les dispositifs d'évaluation et de contrôle du travail et de la vie des enseignants sont de plus en plus fermes (Giroux, 1992 ; Little \& McLaughlin, 1993). Le discours scientifique construit une logique de professionnalisation, qui dépend de l'action menée par les experts eux-mêmes à l'intérieur de leurs espaces académiques et universitaires (Apple, 1993 ; Popkewitz, 1991). Mais le plus décisif se joue dans la façon dont chacun s'approprie de ces images produites à l'échelle mondiale, tout en les localisant dans l'espace-temps de sa propre intervention professionnelle (Goodson \& Walker, 1991 ; Hargreaves, 1994). Le processus identitaire des enseignants s'élabore dans un jeu de pouvoirs et de contre-pouvoirs entre des images qui sont porteuses de différents projets de la profession enseignante. 
Au milieu de toutes les contradictions et paradoxes, on aperçoit des images qui marquent l'identité féminine de la profession enseignante. Elles sont, peut-être, minoritaires, mais laissent entrevoir la possibilité de vivre autrement la profession et ses rapports avec la vie personnelle. Parallèlement, on constate l'existence de constructions discursives qui insistent sur une définition féminine de l'enseignement.

Le bombardement d'images auquel on assiste aujourd'hui a, tout au moins, l'avantage d'attirer l'attention sur les enseignants et de leur permettre d'affirmer publiquement leurs sensibilités et leurs croyances (Altenbaugh, 1992 ; Cunningham, 1992). Je tiens particulièrement aux images qui s'attachent à redéfinir ce que j'appelle les deux paradigmes perdus de la réflexion en éducation: l'enseignant en tant que personne et l'école en tant qu'organisation. Au milieu d'une profusion d'images contradictoires, on remarque la présence, pour la première fois, d'images publiques des femmes-enseignantes. Est-ce le signe que la lente accomodation des enseignants à une identité féminine est à même d'être accomplie?

La question reste ouverte, suggérant des recherches ultérieures. Pour l'instant, je me suis borné à suivre librement une idée, qui s'est progressivement constituée en argument : d'abord, je me suis arrêté aux années de transition du XIX ${ }^{\mathrm{e}}$ vers le $\mathrm{XX}^{\mathrm{e}}$ siècle, pour souligner l'importance des images publiques (essentiellement masculines) des enseignants à un moment décisif de leur affirmation collective et professionnelle ; ensuite, j'ai regardé des images produites au milieu du $\mathrm{XX}^{\mathrm{e}}$ siècle, et j'ai signalé qu'elles étaient majoritairement féminines et privées, c'est-à-dire qu'elles portaient sur des femmes en situation pédagogique ou scolaire ; finalement, j'ai constaté l'existence, aujourd'hui, d'images publiques et féminines des enseignants, en laissant comme hypothèse qu'on est face à un changement significatif du modèle de professionnalisation des enseignants hérité du XIX ${ }^{\mathrm{e}}$ siècle.

L'ambition de ce texte est modeste. Il ne prétend nullement s'ériger en thèse ou même en schéma interprétatif. Bien au contraire, il se veut un regard libre qui peut 
suggérer de nouveaux éclairages sur la problématique du genre au sein de la profession enseignante. C'est une histoire parmi tant d'autres histoires qui peuvent être racontées sur les images passées (et présentes) des enseignants. Des images qui se dédoublent à l'infini et qui constituent l'un des moyens les plus puissants pour parler de la profession enseignante. 


\section{Références bibliographiques}

ABRAHAM, Ada, ed. (1984). L'enseignant est une personne. Paris: Éditions ESF.

ALTENBAUGH, Richard J. (1992). The Teacher's Voice - A Social History of Teaching in Twentieth Century America. London: The Falmer Press.

APPLE, Michael W. (1988). Teachers \& Texts - A Political Economy of Class \& Gender Relations in Education. New York: Routledge.

APPLE, Michael W. (1993). Official Knowledge - Democratic Education in a Conservative Age. New York: Routledge.

ARAÚJO, Helena C. (1993). The Construction of Primary Teaching as Women's Work in Portugal (1870-1933). London: Ph.D. thesis - University of London.

BUTLER, Judith (1993). Bodies that matter - On the discursive limits of "sex". New York and London: Routledge.

CANNING, Kathleen (1994). Feminist History after the Linguistic Turn: Historicizing Discourse and Experience. Signs - Journal of Women in Culture and Society, 19 (2), pp. 368-404.

CASEY, Kathleen (1993). I Answer With My Life: Life histories of women teachers working for social change. New York: Routledge.

CHARTIER, Roger (1993). Différences entre les sexes et domination symbolique. Annales ESC, 48 (4), pp. 1005-1011.

COHN, Marilyn M. \& KOTTKAMP, Robert B. (1993). Teachers - The Missing Voice in Education. Albany, NY: State University of New York Press.

CUBAN, Larry (1983). How did teachers teach, 1890-1980. Theory into Practice, 22 (3), pp. 159-165.

CUBBERLEY, Ellwood P. (1902). Syllabus of Lectures on the History of Education. New York: The Macmillan Company.

CUNNINGHAM, Peter (1992). Teachers' professional image and the Press 1950-1990. History of Education, 21 (1), pp. 37-56.

FOUCAULT, Michel (1963). Le langage à l'infini. Tel Quel, 15, pp. 44-53.

GIROUX, Henry A. (1992). Border Crossings - Cultural Workers and the Politics of Education. London and New York: Routledge. 
GOODSON, Ivor \& WALKER, Rob (1991). Biography, Identity, and Schooling. London: The Falmer Press.

GORE, Jennifer M. (1993). The Struggle for Pedagogies - Critical and feminist discourses as regimes of truth. New York and London: Routledge.

HAMELINE, Daniel (1986). L'éducation, ses images et son propos. Paris: Éditions ESF.

HARGREAVES, Andy (1994). Changing Teachers, Changing Times: teachers' work and culture in the postmodern age. New York: Teachers College Press.

JOSEPH, Pamela Bolotin \& BURNAFORD, Gail E., eds. (1994). Images of Schoolteachers in Twentieth-Century America. New York: St. Martin's Press.

LITTLE, Judith W. \& McLAUGHLIN, Milbrey W., eds. (1993). Teachers' Work - Individuals, Colleagues, and Contexts. New York: Teachers College Press.

LUKE, Carmen \& GORE, Jennifer, eds. (1992). Feminisms and Critical Pedagogy. New York and London: Routledge.

MAYNARD, Mary \& PURVIS, June, eds. (1994). Researching Women's Lives from a Feminist Perspective. London: Taylor \& Francis.

MEYER, John W.; RAMIREZ, Francisco O. \& SOYSAL, Yasemin N. (1992). World Expansion of Mass Education, 1870-1980. Sociology of Education, 65 (2), pp. 128-149.

MIDDLETON, Sue (1993). Educating Feminists - Life Histories and Pedagogy. New York and London: Teachers College Press.

NOVOA, António (1994). Les enseignants : à la recherche de leur profession. European Journal of Teacher Education, 17 (1/2), pp. 35-43.

NOVOA, António (1995). On History, History of Education, and History of Colonial Education. In A. Novoa, M. Depaepe \& E. Johanningmeier, eds. The Colonial Experience in Education: Historical Issues and Perspectives. Gent: Paedagogica Historica, pp. 23-61.

POPKEWITZ, Thomas S. (1991). A Political Sociology of Educational Reform Power/Knowledge in Teaching, Teacher Education, and Research. New York: Teachers College Press.

TYACK, David \& CUBAN, Larry (1995). Tinkering Toward Utopia - A Century of Public School Reform. Cambridge, Massachusetts: Harvard University Press. 